\title{
Anti-Centromere Antibody Positivity in a Patient with Generalized Morphea
}

\author{
Fumi Miyagawa $^{a} \quad$ Anna Nakajima $^{a}$ Yasuhiro Akai ${ }^{b} \quad$ Hideo Asada $^{a}$ \\ aDepartment of Dermatology, Nara Medical University School of Medicine, \\ Kashihara, Japan; bThe Center for Rheumatic Diseases, Nara Medical University School of \\ Medicine, Kashihara, Japan
}

\section{Keywords}

Generalized morphea $\cdot$ Anti-centromere antibody $\cdot$ Systemic sclerosis

\begin{abstract}
We report the case of a 45-year-old female with generalized morphea (GM), who exhibited positivity for the anti-centromere antibody $(A b)$. She frequently developed multiple sclerotic skin lesions, whose histological findings were compatible with morphea. She demonstrated favorable responses to topical and oral steroids. Cases of $\mathrm{GM}$ associated with systemic sclerosis (SSc)-specific Abs (anti-Scl-70 Ab, anti-centromere Ab, and anti-RNA polymerase III Ab) have rarely been reported. The previously reported GM cases involving anti-SSc-specific Abs exhibited some skin manifestations of SSc, such as nailfold capillary changes. However, our case did not show any signs of SSc or limited cutaneous SSc. More cases are needed to clarify whether GM with SSc-specific Abs leads to SSc.

\section{Introduction}

Morphea, which is also referred to as localized scleroderma, is a distinct disease entity from systemic sclerosis (SSc). Generalized morphea (GM) is a generalized form of morphea, which involves $>4$ indurated plaques of $>3 \mathrm{~cm}$ in diameter and/or $\geq 2$ body sites but does not affect the face or hands [1]. Morphea and GM are commonly associated with anti-ssDNA 
antibodies (Abs) [2], but tests for SSc-specific autoantibodies, such as anti-centromere Abs (ACA), anti-Scl-70 Abs, and anti-RNA polymerase III (RNAP III) Abs, usually produce negative results. We describe the case of a patient with GM who exhibited positivity for ACA but did not display any features of SSc.

\section{Case Report}

A 45-year-old female was referred to our department because skin lesions on her legs became itchy. She had noticed sclerotic areas on her lower extremities 5 years ago and was followed up by the internist. At that time, her laboratory test results showed an elevated antinuclear Abs (ANA) level (1:40 in a discrete speckled pattern) and ACA (index: 42.0, normal range $<10$ ). Indirect immunofluorescence was performed using HEp-2 cells as substrate to detect ANA and enzyme-linked immunosorbent assay was used to detect ACA. A physical examination at our department revealed that she had multiple circumscribed, indurated patches, which exhibited hyperpigmentation and atrophy, on her lower legs (Fig. 1a) and right forearm. The lesions had red borders, indicating that they were active. A biopsy specimen from a leg lesion showed thickened collagen bundles throughout the entire dermis, extending into the subcutis, and lymphocytic perivascular infiltration with scattered eosinophils (Fig. 1b). A diagnosis of GM was made. She did not display Raynaud phenomenon, sclerodactyly, or nailfold capillary changes, which ruled out SSc [1]. Her medical history included cholelithiasis. She did not have a history of organic solvent exposure. Routine blood tests and urinalysis produced normal results, except for an elevated creatinine level $(0.84 \mathrm{mg} / \mathrm{dL}$, normal range: $0.46-$ 0.79). Laboratory investigations yielded positive values for the following Abs: ANA in a discrete speckled pattern (1:80 titer), ACA (index: 40.2$)$, and rheumatoid factor $(16 \mathrm{U} / \mathrm{mL}$ ). Conversely, tests for the following Abs produced negative results: anti-ssDNA, anti-dsDNA, antiSSA/Ro, anti-SSB/La, anti-Scl-70, anti-ribonucleoprotein, anti-Sm, and anti-RNAP III Abs. No visceral organ involvement was detected. The patient was treated with topical corticosteroids, which produced a favorable response. Three months later, she noticed two sclerotic patches with red borders on her right abdomen (Fig. 2). Treatment with $300 \mathrm{mg} /$ day tranilast and topical corticosteroids was initiated, which improved the sclerosis. Six months later, she again noticed multiple new lesions. On examination, she presented with numerous new sclerotic plaques surrounded by red borders on her chest, abdomen, bilateral shoulders, and right upper arm (Fig. 3a, b). New lesions or the extension of existing lesions was seen on her lower legs. Oral prednisolone was initiated at a dose of $15 \mathrm{mg}$, and the patient's skin status markedly improved. The prednisolone was gradually tapered and stopped. No recurrence had been detected after 6 months.

\section{Discussion}

The three types of ANA that are most frequently associated with SSc are ACA, anti-Scl-70 Abs, and anti-RNAP III Abs, which are found in over $50 \%$ of SSc patients [3]. They are highly specific and are generally present exclusively of each other [3]. ACA are the most frequently seen autoantibodies in SSc patients, and ACA positivity is strongly associated with the occurrence of limited cutaneous SSc [3]. Morphea and GM are commonly associated with antissDNA Abs, and the serum level of anti-ssDNA Abs was reported to be higher in patients with GM than in those with morphea [2]. 
Cases of GM associated with SSc-specific Abs have rarely been reported. We previously reported a case of GM involving anti-RNAPIII Abs [4], while a case of GM involving ACA has also been described [5]. Both cases involved nailfold capillary changes, one of the characteristic features of SSc, which indicated that SSc might develop in future $[4,5]$. In contrast, no features of SSc, including Raynaud phenomenon, nailfold capillary changes, and sclerodactyly [1], were seen in the present case of GM involving ACA. In a large study of 245 patients with morphea, including 37 patients with GM, only $1 \mathrm{GM}$ patient each was positive for anti-Scl-70 Ab and ACA, respectively, but there was little information about whether these GM patients developed SSc-specific manifestations, such as Raynaud phenomenon, nailfold capillary changes, or sclerodactyly [6].

It is currently unclear whether cases of GM involving SSc-specific Abs behave differently from cases of GM without SSc-specific Abs and whether the present case illustrates an unusual presentation of GM associated with SSc-specific Abs. More cases need to be studied to clarify these issues.

\section{Statement of Ethics}

Informed consent was obtained from the patient and the work was done according to the Declaration of Helsinki.

\section{Disclosure Statement}

The authors have no conflicts of interest to disclose.

\section{References}

1 Fett N, Werth VP. Update on morphea: part I. Epidemiology, clinical presentation, and pathogenesis. J Am Acad Dermatol. 2011 Feb;64(2):217-28.

2 Falanga V, Medsger TA Jr, Reichlin M. Antinuclear and anti-single-stranded DNA antibodies in morphea and generalized morphea. Arch Dermatol. 1987 Mar;123(3):350-3.

3 Nihtyanova SI, Denton CP. Autoantibodies as predictive tools in systemic sclerosis. Nat Rev Rheumatol. 2010 Feb;6(2):112-6.

4 Miyagawa F, Asada H. Reactivity to anti-RNA polymerase III antibody and nailfold capillary changes in patient with generalized morphea combined with multiple autoimmune disorders. Eur J Dermatol. 2018 Feb;28(1):112-3.

5 Ohmatsu H, Tada Y, Yazawa N, Kadono T, Tamaki K. Generalized morphea positive for anticentromere antibody. Eur J Dermatol. 2008 Nov-Dec;18(6):718-9.

6 Leitenberger JJ, Cayce RL, Haley RW, Adams-Huet B, Bergstresser PR, Jacobe HT. Distinct autoimmune syndromes in morphea: a review of 245 adult and pediatric cases. Arch Dermatol. 2009 May;145(5):545-50. 


\section{Case Reports in Dermatology}

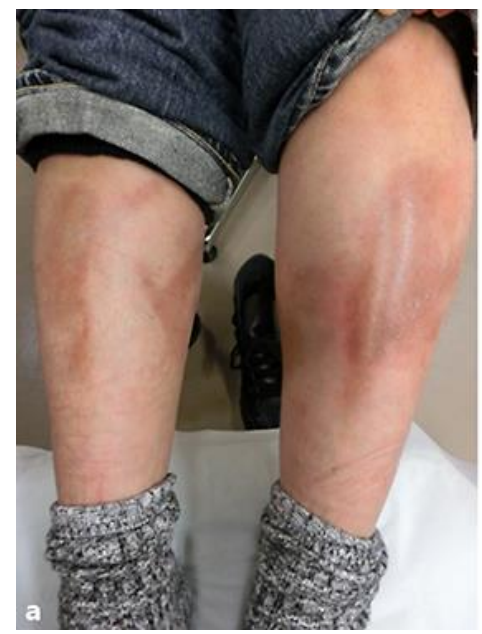

(c) 2018 The Author(s). Published by S. Karger AG, Basel www.karger.com/cde

Miyagawa et al.: Anti-Centromere Ab-Positive Generalized Morphea

Fig. 1. a Sclerotic and atrophic areas were seen on both lower legs. b A histopathological examination revealed dermal and subcutaneous sclerosis with thickened collagen bundles and lymphocytic infiltration (hematoxylin and eosin).

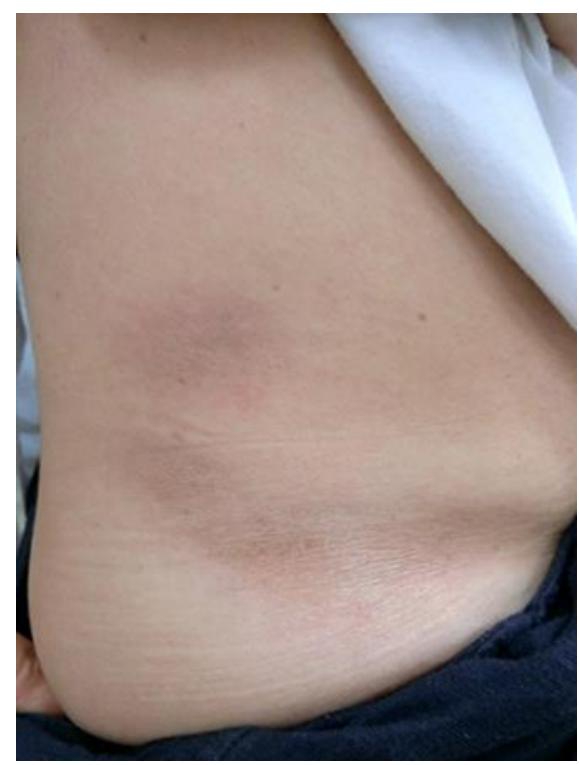

Fig. 2. New sclerotic plaques with violaceous borders appeared on the patient's abdomen. 


\section{Case Reports in Dermatology}

www.karger.com/cde

Miyagawa et al.: Anti-Centromere Ab-Positive Generalized Morphea

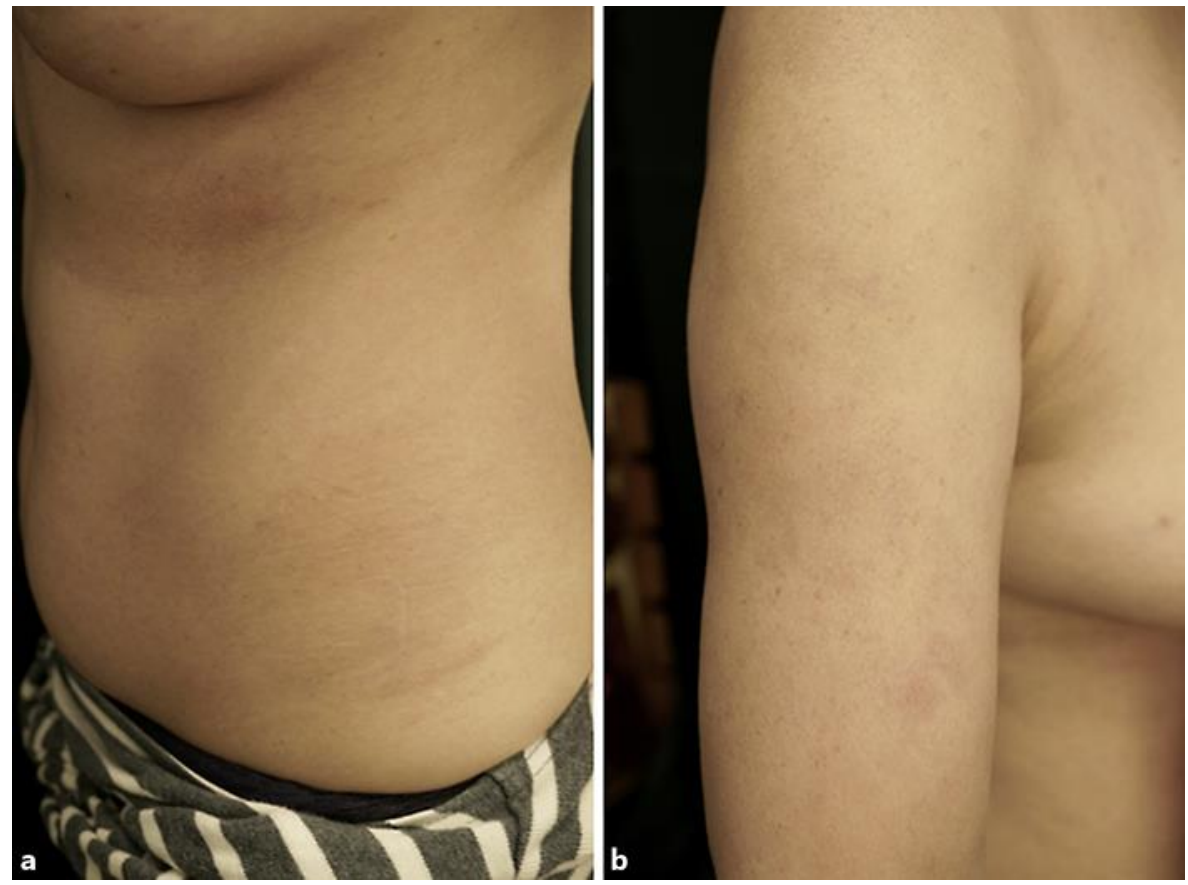

Fig. 3. New sclerotic lesions were widespread on the left trunk (a) and right arm (b). 